\title{
Architecture of optimal transport networks
}

\author{
Marc Durand \\ Matière et Systèmes Complexes \\ UMR 7057 CNRS \& Université Paris 7 - Denis Diderot \\ Tour 33/34 - 2ème étage - case 7056 \\ 2 Place Jussieu - 75251 Paris Cedex 05, France
}

October 31, 2018 


\begin{abstract}
We analyze the structure of networks minimizing the global resistance to flow (or dissipated energy) with respect to two different constraints: fixed total channel volume and fixed total channel surface area. First, we determine the shape of channels in such optimal networks and show that they must be straight with uniform cross-sectional areas. Then, we establish a relation between the cross-sectional areas of adjoining channels at each junction. Indeed, this relation is a generalization of Murray's law, originally established in the context of local optimization. Moreover, we establish a relation between angles and cross-sectional areas of adjoining channels at each junction, which can be represented as a vectorial force balance equation, where the force weight depends on the channel cross-sectional area. A scaling law between the minimal resistance value and the total volume or surface area value is also derived from the analysis. Furthermore, we show that no more than three or four channels meet in one junction of optimal bi-dimensional networks, depending on the flow profile (e.g.: Poiseuille-like or plug-like) and the considered constraint (fixed volume or surface area). In particular, we show that sources are directly connected to wells, without intermediate junctions, for minimal resistance networks preserving the total channel volume in case of plug flow regime. Finally, all these results are illustrated with a simple example, and compared with the structure of natural networks.
\end{abstract}


Networked structures arise in a wide array of different contexts such as water, gas and power supply of a city, vascular systems of plants and animals, or river basins [1] [2]. Thus, optimization of transport in networks has evident industrial and economical importance, but may also shed light on the structure of natural networked structures. Indeed, the analysis of these structures from optimization and selection principles has been recently the subject of intense scientific activity [4 [5] 6] [7] [8] and controversy [9] [10] [11. Besides, theoretical models - based on local optimization (i.e. optimization of the geometry of a single junction) - have been attempted to explain in detail the regular patterns of vascular networks [12] 13] 14]. However, it is generally known that as the global optimum is achieved the local optimum of a single junction is often discarded. In the present paper, we characterize the structure of networks satisfying to the global optimization of transport. For the class of networks mentioned here, euclidean metric must be taken account, and the optimization must be achieved with respect to some geometrical constraint.

Precisely, the problem we consider can be expressed as it follows: consider $s$ sources at the same potential (electrical potential, pressure, concentration, temperature,...) $V_{S}$ and $w$ wells at the same potential $V_{W}$, their respective positions being fixed. What is the architecture of the network linking all the sources to all the wells and minimizing the effective resistance (or dissipated energy), for a fixed total channel volume or fixed total channel surface area [16] ? Or equivalently, which architecture minimizes the total channel volume or surface area for a same value of the global resistance?

In the following, we shall refer often to the electrical circuit terminology, although this study obviously concerns any flow-in-network situation. Let us denote each pipe by a pair of indices $(i, j)$ corresponding to the labels of its two ends. We suppose a priori that pipes can be curved, but we assume that their aspect ratios are sufficiently high so a length $l_{i j}$ and a local cross-sectional area $s_{i j}(l)$ (where $l$ denotes the curvilinear coordinate along a channel) can be unequivocally defined for each pipe $(i, j)$. The resistance $d r_{i j}$ 
of an infinitesimal piece of pipe of length $d l$ is then defined as:

$$
d r_{i j}=\frac{\rho}{s_{i j}^{m}} d l
$$

where $\rho$ is the "resistivity", supposed to be the same for all the pipes. For $m=1$, the flow in each channel is plug-like, while for $m=2$ the flow is Poiseuille-like. Assuming there is no leakage through the pipe lateral surface, the resistance of the whole pipe $(i, j)$ is:

$$
r_{i j}=\int_{0}^{l_{i j}} \frac{\rho}{s_{i j}^{m}} d l .
$$

Since we shall inspect the minimal resistance configuration with respect with two different constraints (a fixed total channel volume $V_{t o t}$ and a fixed total surface-area channel $S_{t o t}$ ), we introduce for simplicity the

"constraint function": $C_{n}=\sum_{(i, j)} \int_{0}^{l_{i j}} s_{i j}^{n} d l$, so that: $C_{1}=V_{t o t}$, and $C_{1 / 2} \propto S_{t o t}$.

\section{Cohn's theorem}

To characterize the architecture of minimal resistance networks, we shall invoke Cohn's theorem, originally developed in the context of electrical circuit analysis [17]: consider a one-port network composed entirely of two-terminal elements with resistances $r_{i j}$. The variation of the effective network resistance $R$ with the variation of the resistance $r_{i j}$ is given by:

$$
\frac{\partial R}{\partial r_{i j}}=\left(\frac{i_{i j}}{I}\right)^{2}
$$

No particular assumption is made on the expression of the resistances $r_{i j}$ for the derivation of this result (indeed, the theorem is still valid for complex impedances). Conservation of flow and energy only are required. Thus, Cohn's theorem can be applied to a broader class of flow-in-network situations. 


\section{Optimal shape of channels}

We first notice that in order for the effective network resistance to be at its minimum value with respect to the constraint $C_{n}$, each channel must be straight with a uniform cross-sectional area (i.e.: $\left.s_{i j}(l)=s_{i j}\right)$. Indeed, we see from eq. 3 that the effective network resistance $R$ is a monotone function of the individual resistances $r_{i j}$. Thus, any small change in pipe diameter or pipe length from the minimal resistance configuration compatible with the constraint - must lead to an increase of the resistances $r_{i j}$. As a consequence, the length of each pipe must be as small as possible and its diameter as large as possible, i.e. each channel must be straight with a uniform cross-sectional area. Besides, it can be noticed that a circular cross-sectional area have the specific property of minimizing both the pipe surface area for a fixed volume (or equivalently maximizing the pipe volume for a fixed surface area) and the dissipative energy in the channel for a fixed incoming flow-rate in case of Poiseuille-flow regime.

\section{Relations between diameters: generalized Murray's law}

We now establish relations between diameters and angles in an optimal network, for a given topology (meaning that no junction or channel can be added or removed from the network, but the channel lengths and cross-

section areas are free to vary). We thus have to minimize the function $\widetilde{R}=R+\lambda C_{n}$ (where $\lambda$ is a Lagrange multiplier) with respect to the independent variables $\left\{s_{i j}\right\}$ and $\left\{\mathbf{r}_{i}=\left(x_{i}, y_{i}\right)\right\}$, respectively the channel crosssectional areas and node positions. Using Cohn's theorem 3 the condition of extremum with respect to the cross-sectional areas $\left(\partial \widetilde{R} / \partial s_{i j}=0\right)$ gives:

$$
\left(\frac{i_{i j}}{I}\right)^{2}=\frac{\lambda}{\rho} \frac{n}{m} s_{i j}^{m+n}
$$


Furthermore, conservation of flow-rate at each junction $i\left(\sum_{j} i_{i j}=0\right)$ implies:

$$
\sum \operatorname{sign}\left(i_{i j}\right) s_{i i}^{(m+n) / 2}=0 .
$$

This relation, illustrated on Fig. 1]: Murray's law 12 to any flow profile the particular case $m=2, n=1) . \mathrm{N}$ optimization of the network structu optimization (flow and channel cross $\mathbf{9}$ was found for a given flow, and not

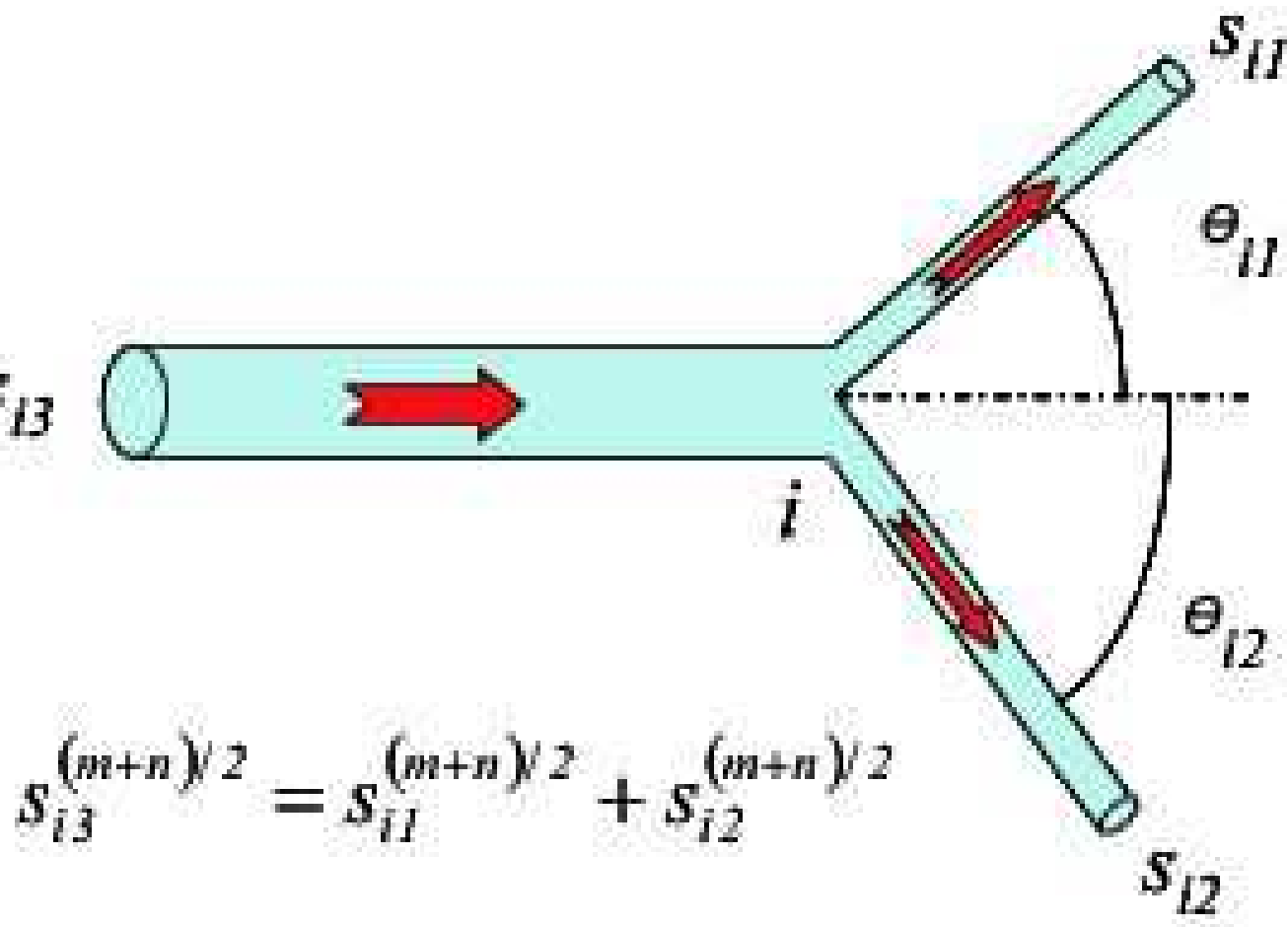

Figure 1: Relation between cross-sectional areas of adjoining channels in a minimal resistance network. This relation is a generalization of Murray's law. 


\section{Geometry of nodes}

Condition of extremum with respect to the node positions $\left(\partial \widetilde{R} / \partial r_{i}=0\right)$ together with relation 4 straightforwardly leads to the following vectorial equality at each node $i$ :

$$
\sum_{j} s_{i j}^{n} \mathbf{e}_{i j}=\mathbf{0}
$$

where $\mathbf{e}_{i j}$ is the outward-pointing unit vector along the channel $(i, j)$ (see Fig. 2). This equality, relating angles between adjoining channels to their cross-sectional areas, is similar to a force balance equation, where the weight of the force acting along the channel $(i, j)$ is directly proportional to $s_{i j}^{n}$. As for Murray's law, local optimization principles have already been proposed in order to describe the geometry of nodes in natural networks, namely: minimization of channel volume (V), channel surface area $(\mathrm{S})$, dissipated power $(\mathrm{P})$, and drag force (D) on the walls [3] [14]. All these approaches consist in varying the position of a given junction, while the positions of the other junctions, the network topology, the channel cross-sectional areas, and the flow-rates through every channel are remained fixed. However, in the context of a global optimization, a change in a node position should alter the flow-rate distribution, and it is therefore to be expected that global minimization of the dissipated energy leads to a different optimal geometry of nodes than in the local optimization context (P). Indeed, the optimal geometry of nodes described by Eq. 6 is similar to the one obtained for (S) (when $n=1 / 2$ ) or (V) (when $n=1$ ), but different from (P) 3] 13].

\section{Scaling-law between minimal resistance and constraint value}

A relation between the minimal resistance value and the constraint value can be established, using Eq. 4 and conservation of energy:

$$
R=\sum_{(i, j)} r_{i j}\left(\frac{i_{i j}}{I}\right)^{2}=\lambda \frac{n}{m} C_{n} .
$$




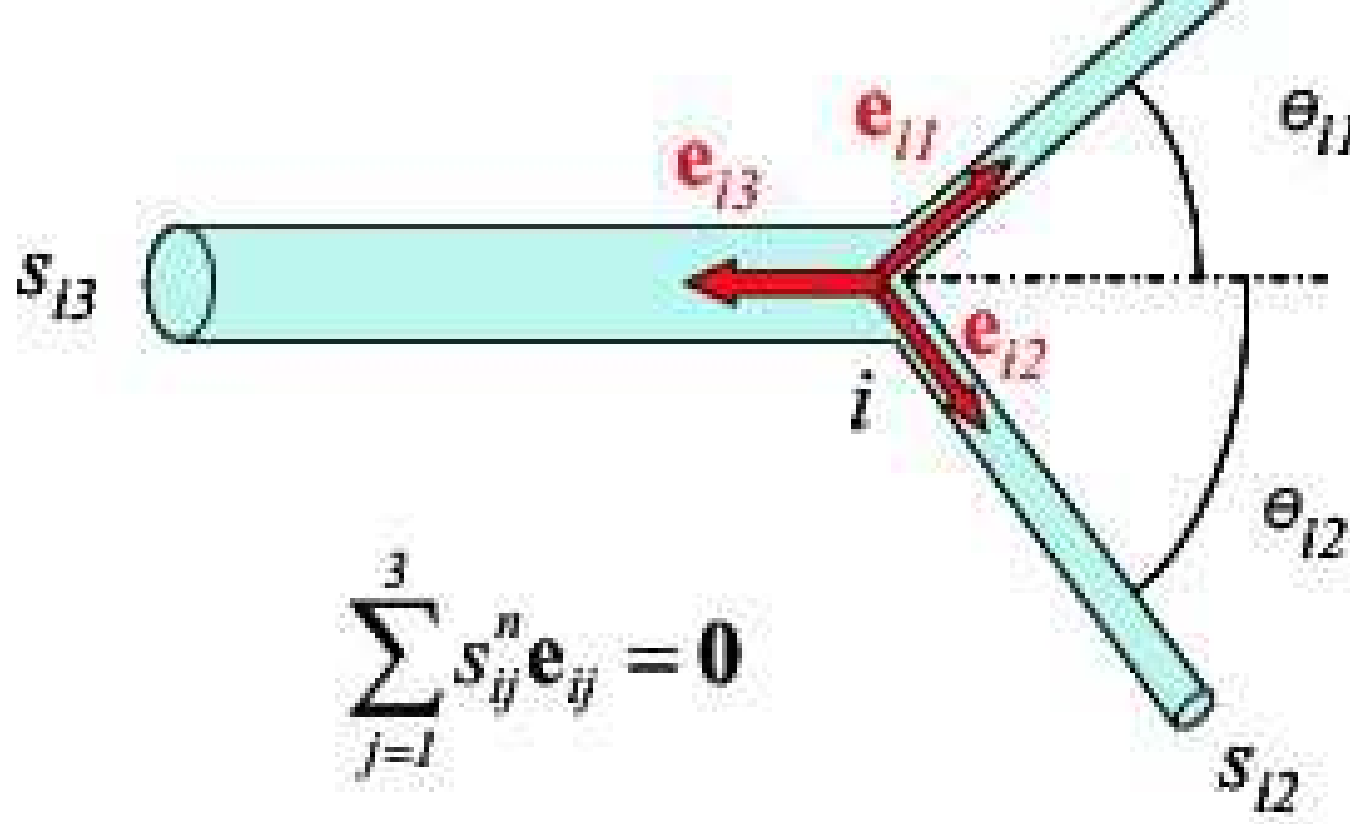

Figure 2: Relation between angles and cross-sectional areas of adjoining channels in a minimal resistance network. This relation is similar to a force balance equation describing the equilibrium of strings tied together and under respective tensions, or weights, $s_{i j}^{n}$.

On the other hand, a classical result of optimization theory relates the Lagrange multiplier to the change of the minimal resistance with respect to the constraint value: $\lambda=-\frac{d R_{m}}{d C n}$ (note that Eq. 7 implies $\lambda \geq 0$ ). Therefore, it is found that the resistance of an optimal network scales as $C_{n}^{-m / n}$, i.e.:

$$
R=\rho l\left(\frac{l}{C_{n}}\right)^{\frac{m}{n}}
$$

where $l$ is a parameter with dimension of length, depending solely on the network topology, the positions of sources and wells, and the values of $m$ and $n$.

We have shown that a minimal resistance configuration, for a given topology, if it does exist, must satisfy to the equations 4 , 5 and 8 Wether the extrema characterized by this set of equations are local minima or local maxima is not clear (although this uncertainty might be dispelled by some convexity argument). Nevertheless, because individual resistances have finite values, there must exist at least one configuration with global minimal resistance (but we do not know if this configuration is unique) [18]. 


\section{Upper bound on the node connectivity}

Finally, we establish an upper bound on the number of channels joining in one node, in a bi-dimensional minimal resistance network. To do so, we look at a given junction of $N$ channels and determine when this junction is preferentially replaced with two junctions respectively of 3 and $N-1$ channels. Suppose we create a new channel of infinitesimal length $d l_{3}$, as depicted in Fig. 3 Then, the length variation of the two other channels joining in the new 3-fold junction are: $d l_{1}=-d l_{3} \cos \theta_{1}$ and $d l_{2}=-d l_{3} \cos \theta_{2}$, with: $\theta_{1}+\theta_{2}=\gamma$, where $\gamma$ is the angle between these two adjacent channels. The variation of the associated resistances are respectively: $d r_{1}=-\rho d l_{3} \cos \theta_{1} / s_{1}^{m}, d r_{2}=-\rho d l_{3} \cos \theta_{2} / s_{2}^{m}$, and $d r_{3}=\rho d l_{3} / s_{3}^{m}$, where $s_{1}, s_{2}$ and $s_{3}$ are the respective channel cross-sectional areas. Moreover, this transformation must preserve the value of $C_{n}$, so the new channel cross-sectional area $s_{3}$ must satisfy:

$$
s_{3}^{n}=s_{1}^{n} \cos \theta_{1}+s_{2}^{n} \cos \theta_{2} .
$$

Using once again Cohn's theorem, we obtain the variation of the effective resistance:

$$
d R=\rho \frac{d l_{3}}{I^{2}}\left(\frac{i_{3}^{2}}{s_{3}^{m}}-\frac{i_{1}^{2} \cos \theta_{1}}{s_{1}^{m}}-\frac{i_{2}^{2} \cos \theta_{2}}{s_{2}^{m}}\right)
$$

Suppose now that the $N$-fold junction was in a minimal resistance configuration. Then, conditions 4 and 6 must be fulfilled, and we can replace $i_{1}^{2}$ and $i_{2}^{2}$ in Eq. 10 by their expressions (Eq. 4). Moreover, conservation of flow rate relates $i_{3}$ to $i_{1}$ and $i_{2}: i_{3}=-i_{1}-i_{2}$. Using Eq. 9] we see that the resistance variation $d R$ is

negative when: $s_{3}^{(m+n) / 2} \geq s_{1}^{(m+n) / 2} \pm s_{2}^{(m+n) / 2}$. The sign in the right-hand side of this inequality is positive when the two adjacent channels are crossed by flows in same direction, and negative when they are crossed by flows in opposite directions. The former inequality can be rewritten as: $\cos \theta_{1}+r^{n} \cos \theta_{2} \geq\left(1 \pm r^{\frac{m+n}{2}}\right)^{\frac{2 n}{m+n}}$, with $r=s_{2} / s_{1}$. Before establishing an upper-bound on the node connectivity, we must notice the following "rules" on the geometry of junctions in optimal networks: 

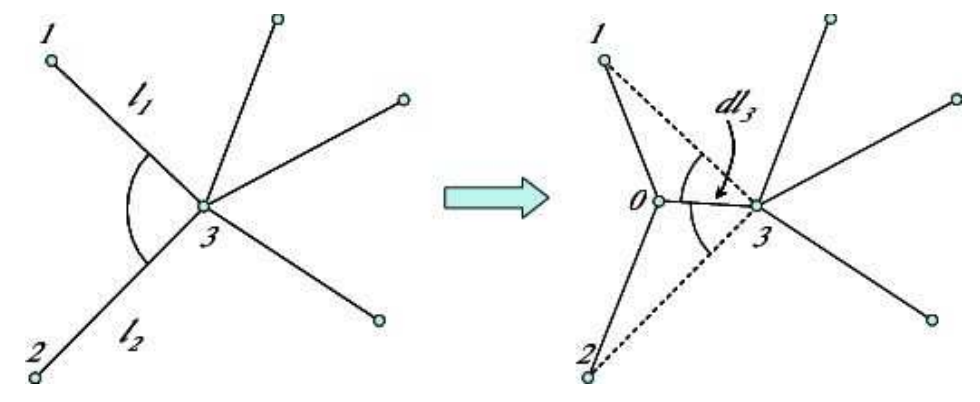

Figure 3: Elementary transformation of a $N$-fold junction to a $(N-1)$-fold junction plus a 3 -fold junction. A new channel, with infinitesimal length $d l_{3}$ is thus created.

1. There is at least one angle lower than $2 \pi / N$ between two adjacent channels in a $N$-fold junction (from geometrical consideration).

2. There is at least one pair of adjacent channels crossed by flows in opposed directions (from flow conservation).

3. The angle between two adjacent channels is always lower than $\pi$ (from Eq. 6).

Let us choose $\theta_{1}$ and $\theta_{2}$ such that $\sin \theta_{1}=r^{n} \sin \theta_{2}$, what corresponds to the maximum value of the left-hand side of the former equality. Since $\gamma \leq \pi$ (rule 3.), we easily check that both $\theta_{1}$ and $\theta_{2}$ are positive and lower than $\pi / 2$, and simple algebra leads to:

$$
\cos \theta_{1}+r^{n} \cos \theta_{2}=\sqrt{1+r^{2 n}+2 r^{n} \cos \gamma}
$$

Thus, the resistance variation $d R$ is negative if and only if:

$$
\cos \gamma \geq f_{ \pm}(r)=\frac{\left(1 \pm r^{\frac{m+n}{2}}\right)^{\frac{4 n}{m+n}}-1-r^{2 n}}{2 r^{n}}
$$

where the functions $f_{+}(r)$ and $f_{-}(r)$ correspond to the respective situations of two adjacent channels crossed by flows in same and opposite directions. The analysis of $f_{+}(r)$ and $f_{-}(r)$ shows that, for any value of $r$, 
these functions are bounded in the following way: $f_{+}(r) \leq 2^{\frac{3 n-m}{m+n}}-1$ for any value of $m, n ; f_{-}(r) \leq 0$ if $m>n$, and $f_{-}(r) \leq-1$ if $m=n$. So if $\gamma$ is lower than $\gamma_{+}=\arccos \left(2^{\frac{3 n-m}{m+n}}-1\right)$ for the first situation, or $\gamma_{-}=90^{\circ}$ (if $m>n$ ) or $180^{\circ}$ (if $m=n$ ) for the second situation, we are ensured that the resistance variation is negative. Let us inspect the different situations:

- If $m=2$, and $n=1 / 2: \gamma_{+} \simeq 97.4^{\circ}, \gamma_{-}=90^{\circ}$. We know there is at least one angle lower than $360^{\circ} / N$ between two adjacent channels in a $N$-fold junction (rule 1.). By choosing this angle as $\gamma$ in the previous analysis, we conclude that a $N$-fold junction is preferably replaced with a $(N-1)$-fold junction plus a 3 -fold junction, as long as $N \geq 4$. The new structure is not in a minimal resistance configuration, determined by Eqs 4 and [6] so the "relaxation" of the new structure such a configuration implies a further decrease of the effective resistance. Eventually, we can repeat the same reasoning on the $(N-1)$-fold junction, if $N-1 \geq 4$. We come to the conclusion that exactly three channels meet at each junction in such an optimal network.

- If $m=2$, and $n=1$ or $m=1$, and $n=1 / 2: \gamma_{+} \simeq 74.9^{\circ}, \gamma_{-}=90^{\circ}$. Following the same argumentation, we conclude that a $N$-fold junction is preferably replaced with a $(N-1)$-fold junction plus a 3 -fold junction as long as $N \geq 5$. Thus, no more than four channels meet in one junction in such an optimized network. Furthermore, it can be noticed that only two kinds of 4 -fold junctions can exist in such a network: either three adjacent channels are crossed by flows of same sign (and the last flow has an opposed sign), or two adjacent channels are crossed by flows with same sign and the two other adjacent channels are crossed by flows with same opposite sign. A 4-fold junction with channels crossed by flows with alternate signs is preferably replaced by two 3-fold junctions, since there is always two adjacent channels crossed by flows with opposite signs and with an angle lower than $90^{\circ}$ (rule 1.).

- If $m=1$ and $n=1: \gamma_{+}=0^{\circ}, \gamma_{-}=180^{\circ}$. But we know that there is always two adjacent channels 
crossed by flows with opposite signs in a $N$-fold junction (rule 2.), with an angle between them lower than $180^{\circ}$ (rule 3 .). So the $N$-fold junction is preferably replaced with a $(N-1)$-fold junction plus a 3-fold junction for any $N \geq 4$. Now, if we let the new structure of the network "relax" to a minimal resistance configuration, it must simultaneously satisfy Eqs. [5] and [6] at every junction, and particularly at the 3 -fold junction. But this set of equations applied in a 3 -fold junction has only trivial solutions when $m \leq n$ : either one cross-section is null, or the three channels are colinear. We conclude that sources are directly connected to the wells, with no intermediate junction, in a minimal resistance network preserving total channel volume and in case of plug-flow regime.

As a concluding remark for this section, we point out that the same reasoning may be used on the total channel length variation instead of resistance variation (Steiner tree problem). In that case, we obtain that links meet at threefold junctions (with equal angles of $120^{\circ}$ ) in a length-minimizing network.

\section{A simple example}

We compare our results with a simple example: two sources and two wells placed at the corner of a rectangle, as depicted on Fig. 4 Four configurations are analyzed. In configuration (1), sources are directly connected to wells, without any intermediate junction. In Configuration (2), sources are connected to wells via a 4-fold intermediate junction. In configurations (3) and (4), sources and wells are connected through two 3 -fold junctions (and the position of intermediate junctions are chosen such that equality [6 is satisfied). The corresponding dimensionless resistance $\frac{R}{\rho a}\left(\frac{C_{n}}{a}\right)^{\frac{m+n}{n}}$ of each configuration is reported on table 1 From these expressions, we note the following observations, in agreement with our results: firstly, we notice that $R$ scales as $\left(1 / C_{n}\right)^{m / n}$. Secondly, when $m=n(=1)$, configuration (1) is the smallest resistance configuration, for any value of the aspect ratio $b / a$. Thirdly, resistance of configuration (3) is always lower than resistance of 
configuration (2) and higher than resistance of configuration (1) $\left(R_{1} \leq R_{3} \leq R_{2}\right)$, for any value of $m, n$, and $b / a$. Fourthly, resistance of configuration (4) is lower than resistance of configuration (2) as soon as: $\sqrt{1+(b / a)^{2}} \leq 2^{2(m-n) /(m+n)}$, for any value of $m, n$, and $b / a$. One can easily check that this criterion on the aspect ratio $b / a$ (for given values of $m$ and $n$ ) corresponds to the condition for Eq. [5] to be simultaneously satisfied with Eq. 6 at each 3-fold junctions of configuration (4). In particular, resistance of configuration

(4) cannot be lower than res:

Fifthly, when $m>n$, resista] sufficiently low value of $b / a$.

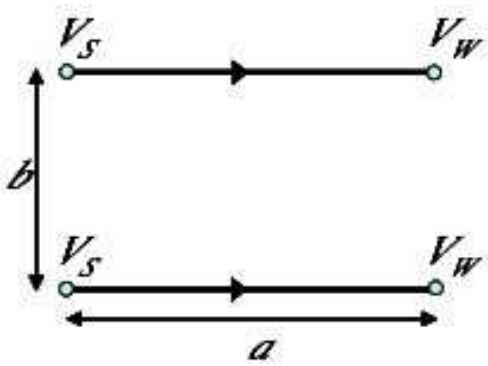

(1)

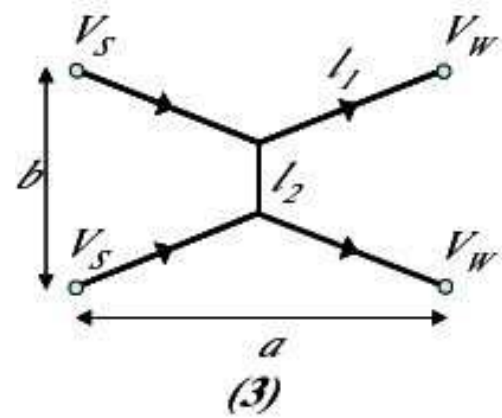

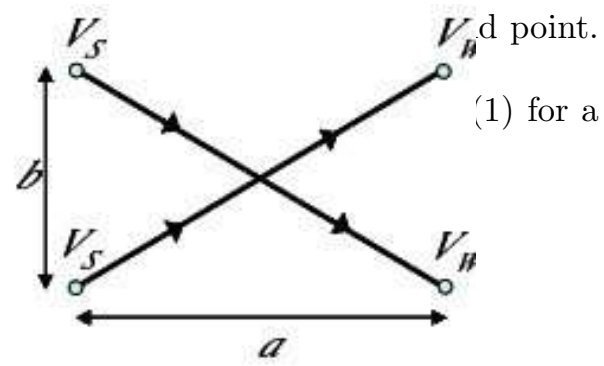

(2)

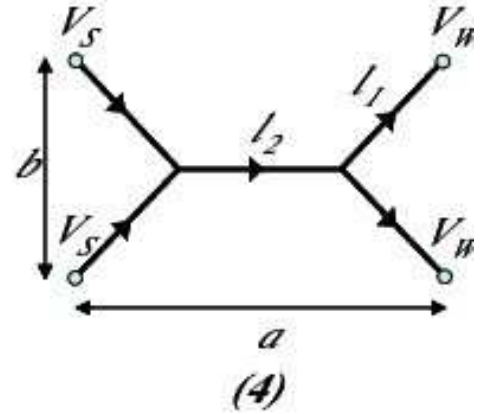

Figure 4: Four different network configurations linking two sources to two wells, placed at the corners of a rectangle of length $a$ and $b$. 


\begin{tabular}{|c|c|}
\hline Configuration & $\frac{R}{\rho a}\left(\frac{C_{n}}{a}\right)^{\frac{m}{n}}$ \\
\hline (1) & $2^{\frac{m-n}{n}}$ \\
\hline (2) & $2^{\frac{m-n}{n}}\left(1+\left(\frac{b}{a}\right)^{2}\right)^{\frac{m+n}{n}}$ \\
\hline (3) & $\begin{array}{l}\widehat{l}_{1}\left(4 \widehat{l}_{1}+2 \widehat{l}_{2} \frac{b / a-\widehat{l}_{2}}{\sqrt{1+\left(b / a-\widehat{l}_{2}\right)^{2}}}\right)^{\frac{m}{n}}, \\
\text { with } \widehat{l}_{1}=\frac{\sqrt{1+\left(b / a-\widehat{l}_{2}\right)^{2}}}{2}\end{array}$ \\
\hline (4) & $\begin{array}{l}\left(4 \widehat{l}_{1}+\widehat{l}_{2} \frac{1-\widehat{l}_{2}}{\widehat{l}_{1}}\right)^{\frac{m}{n}}\left(\widehat{l}_{1}+\widehat{l}_{2}\left(\frac{\widehat{l}_{1}}{1-\widehat{l}_{2}}\right)^{\frac{m}{n}}\right) \\
\text { with } \widehat{l}_{1}=\frac{\sqrt{(b / a)^{2}+\left(1-\widehat{l}_{2}\right)^{2}}}{2}\end{array}$ \\
\hline
\end{tabular}

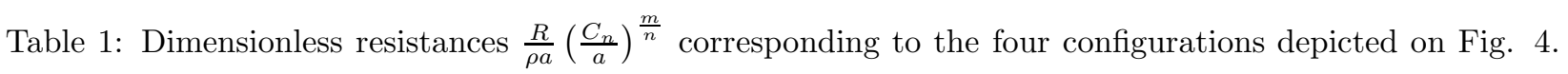
For configurations (3) and (4), $\widehat{l}_{1}=l_{1} / a$ and $\widehat{l}_{2}=l_{2} / a$ are the dimensionless lengths of the two kind of channels.

\section{Comparison with natural networks}

All the results derived in previous sections (relations 4 , 5 6 , 8 as well as the upper-bound on the node connectivity) are consequences of global optimization. However, these results have been established by studying any local perturbation of the structure. Such a local adaptive process may take place during ontogeny of natural networks. Therefore, it may be of interest to compare the structure of some natural networks with the results presented in this work. Indeed, it has been already shown in various publications [19] 20] that Murray's law is well satisfied in some appropriate portions of human and animal vascular systems. In that case, the flow profile is nearly Poiseuille-like $(m=2)$ and the relevant constraint is a fixed total channel volume $(n=1)$ [19]20. Validity of Murray's law for vascular system of plants is more controversial [20] 21] 22] [23], mostly because of the underlying theoretical assumptions in the original derivation of Murray's law, and of the particular structure of veins in vascular system of plants. Nevertheless, 
experimental data suggest that a relation $\sum_{j} \operatorname{sign}\left(i_{i j}\right) s_{i j}^{\nu / 2}=0$ is still verified, with $\nu$ between 2.49 and 3 [15] 23] 25]. Let us look more precisely at the bi-dimensional leaf venation network, like the one reported on Fig. [5. Leaf veins are actually vascular bundles [26, supporting two parallel flows : a pressure-driven flow of water and minerals from petiole to stomata through xylem tissues, and a diffusive flow of nutrients and photosynthesis products in the opposite direction through phloem tissues. So petiole (or major vein) and stomata play alternatively roles of sources and wells for the leaf. An outer layer of cells, called the bundle sheath, surrounds the vascular tissues. Although this layer is not fully impermeable, the leaky radial flow is small when compared with the axial flow, except for the minor veins [21] 27]. For these veins, leakage is very important and the pressure field and nutrient concentration nearby are almost uniform. Kull and Herbig [28] investigated on leaf topology of several species. They observed that leaf venations preferably show trivalent nodes with six neighbors, and noticed that this geometry is typical of self-generating structures like bubble floats. In a recent study, S. Bohn et al. 29] analyzed geometry of junctions in the leaf venation of various species. They observed that angles between veins are very well defined and that a vectorial balance equation comparable to eq. [6 can be established, where the weight of each vector is directly proportional to the vein radius (i.e. $n=1 / 2$ ). Comparison of Bohn et al. observations with our optimization principles suggest then that structure of leaf venation corresponds to a minimization of the resistance for a fixed total channel surface area (or minimization of surface area for a fixed value of resistance). This result is coherent with the idea of a predominant building cost of the bundle sheath cells over those of the vascular tissues [21] 23. Taking $n=1 / 2$ and comparing Eq. 5 with experimental studies of Murray's law leads to a value of $m$ between 1.99 and 2.5, meaning that flow in veins is nearly Poiseuille-like. Note it is assumed in the theory that all channels have same resistivity $\rho$. However, density of xylem and phloem tissues in a leaf vein might be a function of the vein diameter as well. This variation of the resistivity is then include in the coefficient $m$, what could explain the slight difference observed between the experimental value of $m$ and the theoretical 
value for a Poiseuille-flow regime. All these results and the presence of trivalent nodes suggest then that structure of leaf venation correspond to minimal resistance configuration preserving the total surface area and nearly Poiseuille flow profile. The measure of the scaling-law between the hydraulic resistance and the total channel volume or surface area might be an additional way to test this conjecture.

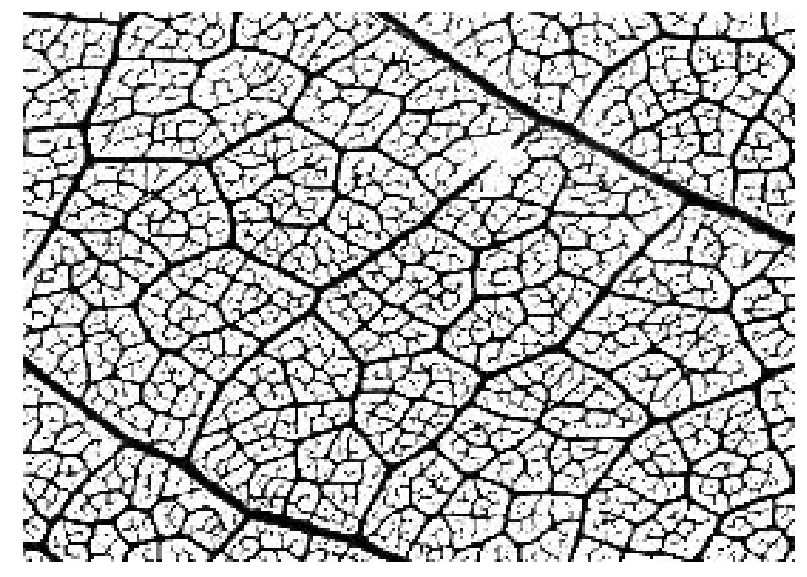

Figure 5: Portion of leaf venation. In most species, the structure is netted-like, and veins meet in 3-fold junctions.

\section{References}

[1] A. Bejan, Rev. Gén. Therm. 36, 592-604 (1997).

[2] S. Lorente, W. Wechsatol, and A. Bejan, Int. J. Heat and Mass Transfer 45, 3299-3312 (2002).

[3] M. A. Changizi and C. Cherniak, Can. J. Physiol. Pharmacol. 78, 603-611 (2000).

[4] G. B. West, J. H. Brown, and B. J. Enquist, Science 276, 122-126 (1997).

[5] G. B. West, J. H. Brown, and B. J. Enquist, Nature 400, 664-667 (1999). 
[6] J. R. Banavar, F. Colaiori, A. Flammini, A. Maritan, and A. Rinaldo, Phys. Rev. Lett. 84, 4745-4748 (2000).

[7] V. Colizza, J. R. Banavar, A. Maritan, and A. Rinaldo, Phys. Rev. Lett. 92, 198701 (2004).

[8] J. R. Banavar, A. Maritan, and A. Rinaldo, Nature 399, 130-132 (1999).

[9] J. Kozlovski and M. Konarzewski, Functional Ecology 18, 283-289 (2004).

[10] J. Whitfield, Nature 413, 342-344 (2001).

[11] J. R. Banavar, J. Damuth, A. Maritan, and A. Rinaldo, Nature 420, 626 (2002).

[12] C. D. Murray, Proc. Natl. Acad. Sci. USA 12, 207-214 (1926).

[13] M. Zamir, J. theor. Biol. 62, 227-251 (1976)

[14] M. Zamir, J. Gen. Physiol. 72, 837-845 (1978).

[15] W. Zhi, Z. Ming, and Y. Qi-Xing, J. Theor. Biol. 209, 383-394 (2001).

[16] Note that a fixed total length is not a pertinent constraint, unless channel cross-sectional areas are bounded; otherwise one could built channels with infinitely large cross-sections and the minimal resistance value would be trivialy zero.

[17] R. M. Cohn, Proc. Am. Math. Soc. 1, 316 (1950).

[18] It is also obvious that there are no global maximum, since one can built a network with infinitely long and indefinitely thin channels (so that value of $C_{n}$ is preserved) and whose resistance is infinitely large.

[19] T. F. Sherman, J. Gen. Physiol. 78, 431-453 (1981).

[20] M. LaBarbera, Science 249, 992-1000 (1990). 
[21] A. Roth-Nebelsick, D. Uhl, V. Mosbrugger, and H. Kerp, Annals of Botany 87, 553-566 (2001).

[22] M. J. Canny, Phil. Trans. R. Soc. Lond. B 341, 87-100 (1993).

[23] K. A. McCulloh, J. S. Sperry, and F. R. Adler, Nature 421, 939-942, (2003).

[24] P. Kruszewski and S. Whitesides, J. Theor. Biol. 191, 221-236 (1998).

[25] N. Kizilova, ICCSA 2004, LNCS 3004, 476-485 (2004).

[26] A. H. de Boer and V. Volkov, Plant, Cell and Environment 26, 87-101 (2003).

[27] M. A. Zwieniecki, P. J. Melcher, C. K. Boyce, L. Sack, and N. M. Holbrook, Plant, Cell and Environment 25, 1445-1450 (2002).

[28] U. Kull and A. Herbig, Naturwissenschaften 82, 441-451 (1995).

[29] S. Bohn, B. Andreotti, S. Douady, J. Munzinger, and Y. Couder, Phys. Rev. E 65, 061914 (2002). 